\title{
The Use of PCA3 Can Reduce the Number of Prostate Biopsies Performed in a Community-Based Urologic Practice
}

\author{
Bradley M. Boelkins ${ }^{1}$, Christopher M. Whelan ${ }^{1,2}$, Richard J. Kahnoski ${ }^{1,2}$, \\ Jonathan Todd Bolthouse ${ }^{1,2}$, Brian R. Lane ${ }^{1,2^{*}}$ \\ ${ }^{1}$ Michigan State University, East Lansing, USA \\ ${ }^{2}$ Spectrum Health Hospital System, Grand Rapids, USA \\ Email: "blaneppmd@yahoo.com
}

Received April 28, 2013; revised May 26, 2013; accepted June 2, 2013

Copyright (C) 2013 Bradley M. Boelkins et al. This is an open access article distributed under the Creative Commons Attribution License, which permits unrestricted use, distribution, and reproduction in any medium, provided the original work is properly cited.

\begin{abstract}
Purpose: It is now generally accepted that PSA screening identifies many prostate cancers that are low-risk and may not need treatment. PCA3 is a prostate cancer-specific marker with improved diagnostic accuracy when compared with PSA in research studies. It remains unknown whether PCA3 testing can reduce the unnecessary diagnosis and treatment of prostate cancer in routine practice. We evaluated whether the use of PCA3 in clinical practice decreases the number of biopsies being performed in response to PSA testing. Methods: 64 consecutive patients undergoing PCA3 measurement in a single community-based urology practice were analyzed for rates of biopsy performance and prostate cancer detection. Results: Median PSA was 5.0 (range: 0.4 - 38.6) and 50\% had undergone prior biopsy without evidence of cancer. Median PCA3 score was 13.6 (range: 1.6 - 133.0) with 14 patients having an elevated PCA3 ( $>35$ ). Prostate biopsy was avoided in 50 of 64 patients $(78 \%)$. Of the 11 patients undergoing biopsy for abnormal PCA3, 7 had prostate cancer (64\%). At $>2$-year median follow-up, 39 of the remaining 50 patients (78\%) avoided subsequent biopsy. Only 5 prostate cancers were diagnosed during follow-up. Conclusions: When used in routine clinical practice, PCA3 appears to reduce the number of biopsies being performed in response to elevated PSA. Given the increasing interest in strategies to reduce unnecessary prostate cancer diagnosis and treatment, this FDA-approved and widely-available molecular test appears to achieve these goals. Further testing will clarify the role of PCA3 in initial and subsequent prostate cancer screening paradigms.
\end{abstract}

Keywords: Prostate Cancer; Prostate Cancer Antigen 3 (PCA3); Prostate Biopsy

\section{Introduction}

Current controversy over PSA screening has arisen from an associated increase in both prostate cancer diagnosis and treatment. The latest United States Preventive Services Task Force (USPSTF) Recommendation Statement has specifically cited the following concerns: lack of direct effect on prostate-cancer specific mortality or overall mortality, the harms of potentially-unnecessary diagnostic procedures (prostate biopsy, PB), and the harms of potentially-unnecessary treatment [1]. Among men with PSA levels $>4 \mathrm{ng} / \mathrm{mL}$ who undergo 10 - 14 core PB, $60 \%-73 \%$ are found not to have cancer on pathologic examination [2-4]. The procedure can cause discomfort and complications, including urosepsis, acute urinary re-

${ }^{*}$ Corresponding author. tention, and hematuria, and the probability of having a positive biopsy decreases with each subsequent biopsy $[5,6]$. These difficulties have motivated development of new and more specific screening tests for prostate cancer to decrease the number of unnecessary PBs.

The PCA3 gene, which is highly over-expressed in prostatic cancer cells, has been found to be more specific for prostate cancer detection than PSA [7-9]. Goode et al. demonstrated that PCA3 had a specificity of 79\% compared to $47 \%$ for PSA for cancer detection at initial biopsy [10]. At repeat biopsy, this disparity in specificity increased to $70 \%$ vs $27 \%$ [10]. PCA3 may therefore find a role in patients prior to initial biopsy and in those who may be considered for repeat biopsy. We hypothesized that urinary PCA3 testing may serve to decrease the number of unnecessary PBs performed in these patients. 
In the present study, we analyzed the utility of PCA3 testing in reducing $\mathrm{PB}$ in a community-based urologic practice.

\section{Materials and Methods}

All patients referred for abnormal PSA and/or digital rectal examination (DRE) were educated regarding the risks and benefits of PSA screening and options for subsequent evaluation. Patients were offered immediate $\mathrm{PB}$ if it was felt that their current risk of $\mathrm{PC}$, and high-grade $\mathrm{PC}$ in particular, warranted this intervention [11]. For patients with a lesser current risk of high-grade PC, discussion included risks and benefits of urinary PCA3 testing to clarify the current risk of prostate cancer. $\mathrm{Pa}-$ tients electing to undergo PCA3 testing gave a postdigital exam urine sample, which was adequate for analysis in $>96 \%$ of cases (Bostwick Laboratories, Richmond, VA; Dianon Systems, Shelton, CT) [12]. The PCA3 value is defined as the ratio of PCA3 mRNA to PSA mRNA multiplied by 1000 . PB was recommended to patients with abnormal PCA3 and not recommended in patients with normal PCA3. For the purposes of our study, abnormal PCA3 was defined a priori as $\geq 35$, according to the manufacturer's instructions. Subsequent prostate cancer screening was performed with annual rectal exam and periodic PSA testing. For patients with elevated future risk of $\mathrm{PC}$, discussion included risks and benefits of prostate cancer chemoprevention [11]. Men with benign prostatic enlargement and/or lower urinary tract symptoms were advised to consider the use of $5 \alpha$ reductase inhibitors (5ARI's).

IRB approval (IRB\#2012-148) was obtained for evaluation of the medical records of all patients in whom urinary PCA3 testing was obtained. Those with a prior history of prostate cancer were excluded and the initial PCA3 test was used as the referent for those with multiple tests. Between October 2009 and June 2012, 64 total patients met inclusion criteria. Data were maintained in a HIPAA-compliant database, which included information pertaining to the decision to perform PCA3 testing and subsequent urologic follow-up in each patient. For statistical comparisons, the level of significance was defined as $\mathrm{p}<0.05$.

\section{Results}

Characteristics of the study population are found in Table 1. Sixty-two of 64 samples were adequate for PCA3 testing (96.9\%). Median PCA3 was 13.6 (IQR: 5.9 - 32.9), with $22 \%$ abnormal (>35). Median PSA value was 5.0 ng/ml (IQR: 3.7 - 6.6) and 13\% had an abnormal DRE. $50 \%$ of men had undergone prior PB, with 13,10 , and 9 men undergoing 1, 2, and 3 or more prior $\mathrm{PB}$, respectively.
Table 1. Clinical information regarding 64 patients evaluated with PCA3*.

\begin{tabular}{cc}
\hline $\begin{array}{c}\text { Median patient age, } \\
\text { yrs (IQR) }\end{array}$ & $64(58-71)$ \\
Percentage of & \\
Caucasians (No.) & $95 \%(61)$ \\
Percentage of & \\
African-Americans (No.) & $5 \%(3)$ \\
Median PSA, ng/ml (IQR) & $5.0(3.7-6.6)$ \\
Percentage with & $13 \%(8)$ \\
abnormal DRE (No.) & \\
Median prostate size, & $38(30-50)$ \\
gm (IQR) & \\
Percentage with prior & $27 \%(17)$ \\
medical therapy for BPH (No.) & \\
Percentage undergoing prior \\
prostate biopsy (No.) & $50 \%(32)$ \\
& $1: 13$ \\
Number of prior & $2: 10$ \\
biopsies (No.) & $3: 2$ \\
& $4: 5$ \\
PCA3 (>35) (No.) & $5: 1$ \\
Median AUA-SS (IQR) & $9: 1$ \\
Median PCA3, (IQR) & $7(3-10)$ \\
Percentage of abnormal & $13.6(5.9-32.9)$ \\
\hline
\end{tabular}

${ }^{*}$ Two patients had a non-informative PCA3.

Median estimated prostate size on DRE was 38 grams (IQR: 30 - 50). A positive correlation was found between PSA level and prostate volume $\left(\mathrm{r}^{2}=0.202, \mathrm{p}=0.0001\right)$. No correlation was demonstrated between PCA3 and PSA levels $\left(\mathrm{r}^{2}=0.0039, \mathrm{p}=0.31\right)$ or between PCA3 level and prostate volume $\left(\mathrm{r}^{2}=0.0007, \mathrm{p}=0.42\right)$. PSA, DRE and biopsy results in patients with normal and abnormal PCA3 values are indicated in Table 2.

An initial decision for prostate biopsy was made in only $22 \%(n=14)$ of men undergoing PCA3 testing, including 11 with abnormal PCA3 values. Three patients with abnormal PCA3 values have not undergone initial biopsy despite this finding. Two of these patients refused biopsy and one elected for active surveillance due to age and multiple prior negative biopsies. Three additional patients elected PB in the absence of an abnormal PCA3, including two with inadequate $\mathrm{PCA} 3$ results and one with ongoing concern about the risk of PC despite normal PCA3. Initial biopsy was therefore not performed in $78 \%$ of patients (n $=50$ ). Median PCA3 for patients undergoing or not undergoing PB was 52.2 (IQR: 44.6 - 63.8) vs 8.5 (IQR: 5.3 - 20.6), respectively ( $<<0.001)$. Median PCA3 for patients with or without cancer at PB was 55 (IQR: 51 - 88) and 44 (IQR: $43-48)$, respectively $(\mathrm{p}=0.15)$. 
Table 2. Initial PB results according to PCA3.

\begin{tabular}{|c|c|c|}
\hline & Abnormal PCA3 $(n=14)$ & Normal PCA3 $(n=50)$ \\
\hline Median PSA, ng/ml (IQR) & $5.1(4.4-6.2)$ & $5.0(3.6-7.4)$ \\
\hline Percentage with abnormal DRE (No.) & $0 \%(0)$ & $16 \%(8)$ \\
\hline Proportion in whom PB recommended (No.) & $100 \%(14)$ & $0 \%(0)$ \\
\hline Proportion in whom PB performed (No.) & $79 \%(11)$ & $6 \%(3)$ \\
\hline Proportion with PC (No.) & $50 \%(7)$ & $2 \%(1)$ \\
\hline - Gleason 6 & 5 & 0 \\
\hline - Gleason 7 & 1 & 1 \\
\hline • Gleason 8 or higher & 1 & 0 \\
\hline Proportion with primary Gleason pattern 4 or 5 (No.) & $14 \%(2)$ & $0 \%(0)$ \\
\hline Proportion with clinically-insignificant PC (No.) & $14 \%(2)$ & $0 \%(0)$ \\
\hline Median number of cores involved (range) & $3(1-12)$ & 3 \\
\hline \multicolumn{3}{|l|}{ Treatment Modality } \\
\hline Surveillance & 3 & 0 \\
\hline Brachytherapy & 1 & 1 \\
\hline Robotic prostatectomy & 2 & 0 \\
\hline Androgen-deprivation & 1 & 0 \\
\hline
\end{tabular}

${ }^{*}$ Clinically-insignificant PC defined as Gleason score no higher than $6,1-2$ cores positive, $50 \%$ or less cancer in any involved core, and PSA density $<0.15$ [13].

In patients with abnormal PCA3, cancer was detected at initial $\mathrm{PB}$ in 7 patients, which represents $64 \%$ of $\mathrm{PB}$ performed for abnormal PCA3 and 50\% of patients with abnormal PCA3 overall (Table 2). Predominant Gleason pattern 4 was present in 2 patients (Gleason $4+3$ and $4+$ 5), with Gleason $3+3$ in the remaining 5 patients, including 2 clinically-insignificant cancers (Gleason $3+3$ in $<50 \%$ of $1-2$ cores and PSA density $<0.15$ ) [13]. Initial active treatment was pursued in 4 patients and surveillance in 3 patients. Cancer was detected at initial PB in 1 of 3 patients with normal PCA3 values. This patient elected brachytherapy for Gleason $3+4$ cancer detected in 3 of 13 cores.

With median 25 month follow-up (IQR: 14 - 29 months) after PCA3 testing, $81 \%$ of those with normal PCA3 have undergone follow-up DRE and $72 \%$ have undergone subsequent PSA testing. The median follow-up PSA value was 4.3 (IQR: 2.0 - 6.4). The median decrease in PSA in patients taking or not taking $5 \alpha$-reductase inhibitors was $25 \%$ (IQR: $0 \%-52 \%$ ) vs $0 \%$ (IQR: $-4 \%$ $6 \%)$, respectively $(\mathrm{p}=0.005)$. Two patients died in follow-up, both from non-prostate cancer-related issues. $78 \%$ of patients $(n=39)$ have not undergone subsequent biopsy (Table 3). Indications for PB during follow-up included rising PSA $(n=9)$, new prostate nodule on DRE $(\mathrm{n}=1)$, and abnormal repeat PCA3 $(\mathrm{n}=1)$. PC was detected in $45 \%$ of these 11 patients $(\mathrm{n}=5)$, each having a predominant Gleason pattern of 3. PC detected in follow-up included 2 clinically-insignificant cancers according to Epstein criteria [13]. Treatment selection included active surveillance $(\mathrm{n}=3)$ and robotic prostatectomy $(\mathrm{n}=$ 2).
Table 3. Follow-up information at 25 months after initial normal PCA3 screening $(n=50)$.

\begin{tabular}{cc}
\hline Proportion in whom PB was recommended (No.) & $22 \%(11)$ \\
\hline Indication for follow-up biopsy & \\
$\bullet \quad$ Rising PSA & 9 \\
- New prostate nodule & 1 \\
Abnormal PCA3 at repeat testing & 1 \\
Proportion with PC & $10 \%(5)$ \\
• Gleason 6 & 3 \\
- Gleason 7 & 2 \\
Proportion with primary Gleason & 0 \\
pattern 4 or 5 (No.) & $0 \%(0)$ \\
Proportion with clinically-insignificant PC ${ }^{*}$ (No.) & $40 \%(2)$ \\
Median number of cores involved (range) & $2(2-9)$ \\
Treatment Modality & \\
• Surveillance & 2 \\
• Brachytherapy & 0 \\
Robotic prostatectomy & 3 \\
Androgen-deprivation & 0 \\
\hline
\end{tabular}

${ }^{*}$ Clinically-insignificant PC defined as Gleason score no higher than 6, 1 - 2 cores positive, $50 \%$ or less cancer in any involved core, and PSA density $<0.15$ [13].

\section{Discussion}

The weaknesses of PSA as a screening tool for prostate cancer are under intensifying scrutiny within the changing landscape of prostate cancer diagnosis and treatment. [1] PSA, a prostate tissue specific (rather than prostate cancer specific) assay, was first approved by the Food and Drug Administration (FDA) in 1986 for prostate cancer surveillance and in 1994 as a screening tool in 
asymptomatic men [14-16]. During the last two decades, widespread PSA testing in the United States has greatly increased the incidence of PC as more men are subjected to prostate biopsy. Indeed, three fourths of US men 50 years of age and older underwent a PSA test [17]. The poor specificity and sensitivity of PSA have been outlined in multiple studies, including the Prostate Cancer Prevention Trial $[17,18]$. PSA threshold values of 1.1 , $2.1,3.1$, and $4.1 \mathrm{ng} / \mathrm{mL}$ yielded sensitivities of $83.4 \%$, $52.6 \%, 32.2 \%$, and $20.5 \%$ and specificities of $38.9 \%$, $72.5 \%, 86.7 \%$ and $93.8 \%$ respectively [18]. The authors concluded that there is no threshold PSA with both adequate sensitivity and specificity for healthy men [18]. This clouds the decision-making process for the patient, primary care physician and urologist in the interpretation of PSA values.

Even so, over 1 million prostate biopsies are performed annually among Medicare beneficiaries, largely in response to screening with PSA [19]. Recently, Loeb and associates published data on a cohort of 17,472 men from the SEER-Medicare database that underwent prostate biopsy [19]. The authors found that prostate cancer was diagnosed in only $17 \%$ of men. In addition, $6.9 \%$ of men were hospitalized within 30 days of biopsy, more than twice the proportion in a control population [19]. While often viewed as a routine office procedure, prostate biopsy offers a real risk of bleeding and infection in patients with an elevated PSA, the majority of who do not have prostate cancer.

The problems with PSA as a screening test have been emphasized by the US Preventative Services Task Force and the American Academy of Family Physicians who recommend against routine PSA screening to detect prostate cancer in asymptomatic men [1]. While this remains the subject of intense debate within the medical community, most would agree the development of a more accurate screening tool for prostate cancer is critical.

Discovered in 1999, PCA3 is a prostate specific gene that is over expressed in $95 \%$ of prostate cancer cells, at levels that are a median 66-fold higher than adjacent noncancerous prostate cells [7-9]. A commercially-available PCA3 assay has been approved as an adjunct to PSA testing in men with a suspicion of prostate cancer. Unlike PSA, PCA3 is not significantly influenced by age, inflammation, the use of 5-alpha reductase inhibitors or $\mathrm{BPH}$. Gaining ground in academic institutions, PCA3 has been shown to have improved specificity as compared to PSA alone. A large multicenter trial recently evaluated the diagnostic performance of PCA3 to detect PC in men with increased PSA. Men underwent both PSA and PCA3 testing prior to planned PB in response to increased PSA $(2.5 \mathrm{ng} / \mathrm{ml}$ or greater) and/or abnormal DRE. The vast majority of urine samples $(97.5 \%)$ were adequate for PCA3 testing and $33.4 \%$ of these yielded a
PCA3 score $>35$ [20]. Using this cutpoint for PCA3, the number of false-positives (unnecessary biopsies) was reduced by $77 \%$. Crawford and colleagues concluded that "urinary PCA3 testing in conjunction with PSA has the potential to significantly decrease the number of unnecessary prostate biopsies" [20].

Herein, we describe the actual impact of urinary PCA3 testing on the number of PBs performed in a communitybased urology practice. We report on a series of patients in whom PCA3 was measured in lieu of a decision for immediate PB based on PSA alone. We found that PB was avoided in $78 \%$ of patients that underwent initial PCA3 testing, almost identical to that predicted by Crawford et al. [20]. In addition, at a median follow-up of 25 months, PB was avoided in $78 \%$ of men who were followed after a normal PCA3 value. In men who underwent PB for an abnormal PCA3, prostate cancer was detected in $64 \%$, similar to the $61 \%$ PC detection rate in the prior study [20]. No patient with PCA3 score $<35$ was found to have a predominant Gleason pattern $4 / 5$ cancer, while $18 \%$ of patients undergoing PB for abnormal PCA3 had a predominant Gleason pattern 4.

This study has several limitations, including the absence of pathologic confirmation of the absence of $\mathrm{PC}$ in patients electing not to undergo $\mathrm{PB}$, leaving the false negative rate unknown. While this limitation was absent from other research studies that have compared the performance of PCA3 with PSA and other parameters for prediction of $\mathrm{PC}$ at $\mathrm{PB}$ in men who have already made a decision for $\mathrm{PB}$, the design of those studies prevents an analysis of the impact of PCA3 testing on the decision to pursue PB. While the diagnostic performance of PCA3 has by now been well-categorized, the clinical impact of PCA3 score has not been evaluated. The purpose of our study was to determine the actual impact that introduction of PCA3 may have upon clinical practice; our results indicate a reduction in the number of $\mathrm{PB}$ performed, supporting our hypothesis. Another limitation of the study is a smaller sample size, which was impacted by lack of coverage for PCA3 testing in many patients who otherwise would have undergone testing. These and other selection biases may limit the generalizability of our findings, and a future direction of this research is to collaborate with other sites to further assess the generalizability of our findings.

The current study indicates that PCA3 testing can significantly reduce the number of biopsies performed in response to elevated PSA within a community-based urologic practice. If more community practices adopt this approach, these improvements may be realized on a larger scale. This may ultimately reduce the morbidity associated with the overuse of prostate biopsy. A strategy that leads to the performance of fewer prostate biopsies with an improved PC detection rate when performed will 
greatly improve the quality of $\mathrm{PC}$ care within the community. Previous studies have shown the sensitivity of PCA3 score to be lower than PSA testing, indicating that PCA3 should not replace PSA as a screening tool $[10,12$, $16,17]$. Instead, given its superior specificity, PCA3 score could be used to stratify men with elevated PSA based on risk of PC. The addition of PCA3 score to PSA and other clinical parameters within a nomogram predicting PC and high-grade PC has been proposed and warrants further validation [21].

\section{Conclusion}

Measurement of PCA3 score in a community-based urologic practice successfully reduced the number of prostate biopsies performed in response to PSA testing by $78 \%$. At a median of $>2$ year follow-up of these patients, $78 \%$ of these men also avoided subsequent biopsy. Conversely, PC was detected in $64 \%$ of men undergoing $\mathrm{PB}$ for a PCA3 score $>35$. Measurement of PCA3 score in lieu of PB for men with elevated PSA has the potential to reduce unnecessary prostate cancer diagnosis and treatment if used more widely.

\section{Acknowledgements}

The authors would like to thank Kevin Hinton, Bonnie Dykstra and Maureen Gebben for assistance with research activities.

\section{REFERENCES}

[1] V. A. Moyer, on Behalf of the US Preventive Services Task Force, "Screening for Prostate Cancer: US Preventive Services Task Force Recommendation Statement," Annals of Internal Medicine, Vol. 157, No. 2, 2012, pp. 120-134.

[2] R. J. Babaian, A. Toi, K. Kamoi, P. Troncoso, J. Sweet, R. Evans, et al., "A Comparative Analysis of Sextant and an Extended 11-Core Multisite Directed Biopsy Strategy," Journal of Urology, Vol. 163, No. 1, 2000, pp. 152-157. doi:10.1016/S0022-5347(05)67993-1

[3] C. K. Naughton, D. C. Miller, D. E. Mager, D. K. Ornstein and W. J. Catalona, "A Prospective Randomized Trial Comparing 6 versus 12 Prostate Biopsy Cores: Impact on Cancer Detection," Journal of Urology, Vol. 164, No. 2, 2000, pp. 388-392. doi:10.1016/S0022-5347(05)67367-3

[4] J. C. Presti Jr., J. J. Chang, V. Bhargava and K. Shinohara, "The Optimal Systematic Prostate Biopsy Scheme Should Include 8 Rather than 6 Biopsies: Results of a Prospective Clinical Trial," Journal of Urology, Vol. 163, No. 1, 2000, pp. 163-167. doi:10.1016/S0022-5347(05)67995-5

[5] B. Djavan, V. Ravery, A. Zlotta, P. Dobronski, M. Dobrovits, M. Fakhari, et al., "Prospective Evaluation of Prostate Cancer Detected on Biopsies 1, 2, 3 and 4: When
Should We Stop?" Journal of Urology, Vol. 166, No. 5, 2001, pp. 1679-1683.

doi:10.1016/S0022-5347(05)65652-2

[6] G. I. Pinkhasov, Y. K. Lin, R Palmerola, P. Smith, F. Mahon, M. G. Kaag, et al., "Complications Following Prostate Needle Biopsy Requiring Hospital Admission or Emergency Department Visits-Experience from 1000 Consecutive Cases," BJU International, Vol. 110, No. 3, 2012, pp. 369-374.

doi:10.1111/j.1464-410X.2011.10926.x

[7] M. J. Bussemakers, A. van Bokhoven, G. W. Verhaegh, F. P. Smit, H. F. Karthaus, J. A. Schalken, et al., "DD3: A New Prostate-Specific Gene, Highly Overexpressed in Prostate Cancer," Cancer Research, Vol. 59, No. 23, 1999, pp. 5975-5979.

[8] J. B. de Kok, G. W. Verhaegh, R. W. Roelofs, D. Hessels, L. A. Kiemeney, T. W. Aalders, et al., "DD3(PCA3), a very Sensitive and Specific Marker to Detect Prostate Tumors," Cancer Research, Vol. 62, No. 9, 2002, pp. 2695-2698.

[9] D. Hessels, M. P. van Gils, O. van Hooij, S. A. Jannink, J. A. Witjes, G. W. Verhaegh, et al., "Predictive Value of PCA3 in Urinary Sediments in Determining ClinicoPathological Characteristics of Prostate Cancer," Prostate, Vol. 70, No. 1, 2010, pp. 10-16. doi:10.1002/pros.21032

[10] R. R. Goode, S. J. Marshall, M. Duff, E. Chevli and K. K. Chevli, "Use of PCA3 in Detecting Prostate Cancer in Initial and Repeat Prostate Biopsy Patients," Prostate, Vol. 73, No. 1, 2012, pp. 48-53.

[11] M. J. Roobol, F. H. Schroder, E. D. Crawford, S. J. Freedland, A. O. Sartor, N. Fleshner, et al., "A Framework for the Identification of Men at Increased Risk for Prostate Cancer," Journal of Urology, Vol. 182, No. 5, 2009, pp. 2112-2120. doi:10.1016/j.juro.2009.07.018

[12] J. Groskopf, S. M. Aubin, I. L. Deras, A. Blase, S. Bodrug, C. Clark, et al., "APTIMA PCA3 Molecular Urine Test: Development of a Method to Aid in the Diagnosis of Prostate Cancer," Clinical Chemistry, Vol. 52, No. 6, 2006, pp. 1089-1095. doi:10.1373/clinchem.2005.063289

[13] J. I. Epstein, D. W. Chan, L. J. Sokoll, P. C. Walsh, J. L. Cox, H. Rittenhouse, et al., "Nonpalpable Stage T1c Prostate Cancer: Prediction of Insignificant Disease Using Free/Total Prostate Specific Antigen Levels and Needle Biopsy Findings," Journal of Urology, Vol. 160, No. 6, 1998, pp. 2407-2411.

[14] M. C. Wang, L. A. Valenzuela, G. P. Murphy and T. M. Chu, "Purification of a Human Prostate Specific Antigen," Investigative Urology, Vol. 17, No. 2, 1979, pp. 159-163.

[15] M. Kuriyama, M. C. Wang, C. L. Lee, C. S. Killian, L. D. Papsidero, H. Inaji, et al., "Multiple Marker Evaluation in Human Prostate Cancer with the Use of Tissue-Specific Antigens," Journal of the National Cancer Institute, Vol. 68, No. 1, 1982, pp. 99-105.

[16] W. H. Cooner, B. R. Mosley, C. L. Rutherford Jr., J. H. Beard, H. S. Pond, W. J. Terry, et al., "Prostate Cancer Detection in a Clinical Urological Practice by Ultrasonography, Digital Rectal Examination and Prostate Specific Antigen," Journal of Urology, Vol. 143, No. 6, 1990, pp. 
$1146-1154$.

[17] I. M. Thompson, D. P. Ankerst, C. Chi, M. S. Lucia, P. J. Goodman, J. J. Crowley, et al., "Operating Characteristics of Prostate-Specific Antigen in Men with an Initial PSA Level of $3.0 \mathrm{ng} / \mathrm{ml}$ or Lower," JAMA, Vol. 294, No. 1, 2005, pp. 66-70. doi:10.1001/jama.294.1.66

[18] I. M. Thompson, P. J. Goodman, C. M. Tangen, M. S. Lucia, G. J. Miller, L. G. Ford, et al., "The Influence of Finasteride on the Development of Prostate Cancer," The New England Journal of Medicine, Vol. 349, No. 3, 2003, pp. 215-224. doi:10.1056/NEJMoa030660

[19] S. Loeb, H. B. Carter, S. I. Berndt, W. Ricker and E. M. Schaeffer, "Complications after Prostate Biopsy: Data from SEER-Medicare,” Journal of Urology, Vol. 186, No.
5, 2011, pp. 1830-1834. doi:10.1016/j.juro.2011.06.057

[20] E. D. Crawford, K. O. Rove, E. J. Trabulsi, J. Qian, K. P. Drewnowska, J. C. Kaminetsky, et al., "Diagnostic Performance of PCA3 to Detect Prostate Cancer in Men with Increased Prostate Specific Antigen: A Prospective Study of 1962 Cases," Journal of Urology, Vol. 188, No. 5, 2012, pp. 1726-1731. doi:10.1016/j.juro.2012.07.023

[21] A. K. Wu, A. C. Reese, M. R. Cooperberg, N. Sadetsky and K. Shinohara, "Utility of PCA3 in Patients Undergoing Repeat Biopsy for Prostate Cancer," Prostate Cancer and Prostatic Diseases, Vol. 15, No. 1, 2012, pp. 100105. doi:10.1038/pcan.2011.52 